\title{
Finite-Time Consensus with a Time-Varying Reference State and Switching Topology
}

\author{
Jian-Yong Wang, ${ }^{1}$ Zhen Tian, ${ }^{1}$ Xu Zhu, ${ }^{2}$ and Naif D. Alotaibi ${ }^{3}$ \\ ${ }^{1}$ College of Mechanical Engineering, Liaoning Technical University, Fuxin, Liaoning 123000, China \\ ${ }^{2}$ Ningxia Public Resource Trading Authority, Yinchuan, Ningxia 750002, China \\ ${ }^{3}$ Department of Electrical and Computer Engineering, Faculty of Engineering, King Abdulaziz University, Jeddah, Saudi Arabia
}

Correspondence should be addressed to Jian-Yong Wang; 5446941@qq.com

Received 19 March 2017; Accepted 12 April 2017; Published 1 June 2017

Academic Editor: Chaojie Li

Copyright (C) 2017 Jian-Yong Wang et al. This is an open access article distributed under the Creative Commons Attribution License, which permits unrestricted use, distribution, and reproduction in any medium, provided the original work is properly cited.

\begin{abstract}
The finite-time consensus problem in the networks of multiple mobile agents is comprehensively investigated. In order to resolve this problem, a novel nonlinear information exchange protocol is proposed. The proposed protocol ensures that the states of the agents are converged to a weighted-average consensus in finite time if the communication topology is a weighted directed graph with a spanning tree and each strongly connected component is detail-balanced. Furthermore, the proposed protocol is also able to solve the finite-time consensus problem of networks with a switching topology. Finally, computer simulations are presented to demonstrate and validate the effectiveness of the theoretical analysis under the proposed protocol.
\end{abstract}

\section{Introduction}

In recent years, the cooperative control has received considerable attention mainly due to its broad applications to multiagent systems such as the state consensus seeking of multiple mobile vehicles [1-3], flocking, rendezvous, and containment in natural and social systems [4-9], analysis and control of cyberphysical networks [10], and distributed control and dispatching in smart grids [11-17]. The cooperative control is an interdisciplinary subject and several researchers from different scientific communities have conducted various research studies concerning the abovementioned applications $[2,18]$. Among the numerous research topics in cooperative control, consensus problem is one of the most important issues $[19,20]$. The consensus problem can be generally described as how to design appropriate protocols based on the local information under some communication topology to ensure that the concerned agents reach an agreement on certain quantities of interest.

The main concern of this paper is the consensus seeking problem in the distributed control systems. Consensus seeking problem is a long-standing issue in computer science and sometimes called agreement problem. The communication topology and the consensus protocol are crucial for the consensus of multiagent systems. The researchers have mainly focused on the following two issues: one is the determination of the weakest and the simplest communication topology under which the given protocol can ensure the agents reach an agreement; the second is the design of a protocol to ensure that the states of the agents are converged to consensus under the given interaction topology. The multiagent consensus problem has been addressed by using the graph theory and the matrix analysis methods under various interaction topologies with given information exchange protocols. Vicsek et al. [4] have introduced an interesting discrete-time model of mobile agents. Each agent's motion is updated according to a local rule based on its own and its neighbors states. The theoretical explanation of the consensus property of the Vicsek mode has been introduced in [5], where each agent's set of neighbors should change over time with evolution of the system. Later, a general framework of the consensus problem for networks of dynamics agents with fixed or switching topology and communication time-delays has been established [19]. The consensus conditions derived in [19] have been further relaxed in [20]. Lin et al. [21, 22] have studied the consensus problem in the context of formation control of autonomous vehicles and have demonstrated that formation stabilization to a certain point is only feasible 
if the sensor digraph has a globally reachable node. The consensus problem has also been studied for switching communication topology $[23,24]$, asynchronous consensus $[25,26]$, high-dimensional consensus [27], consensus with sampled communication [28], and consensus with external disturbances and model uncertainties [29]. The research so far has focused more on the determination of the weakest and the simplest communication topology than the design of the information exchange protocol to achieve consensus under the given topology. The convergence rate is a very important performance index to evaluate the protocols. Although the enlargement of the algebraic connectivity can increase the convergence rate but still the protocols are unable to ensure that the states of the agents will reach a consensus in finite time. Finite-time consensus is desirable in many real-time scenarios. Recently, Wang and Xiao have studied the finitetime consensus problems and provided several finite-time consensus protocols to ensure that the multiagents reach a consensus in finite time with appropriate communication topology [30-32]. Finite-time consensus in multiple EulerLagrange systems over undirected topology has been studied in [33]. More recently, finite-time consensus tracking problem of multiple high-order systems has been considered in $[34,35]$. In this paper, a new nonlinear protocol based on the abovementioned works is proposed. The proposed protocol ensures that the agents reach the finite-time consensus with a better convergence rate than the typical linear protocol [19] and the nonlinear protocol proposed by Feng and Long [30]. Furthermore, a number of sufficient consensus conditions are derived and discussed using the tools from Lyapunov stability analysis. Finally, numerical examples are provided to validate the analytical results.

The remainder of the paper is organized as follows: in Section 2, some preliminary notions in graph theory are provided. Then, the problem is formulated in Section 3. Theoretical analysis results are given in Section 4. In Section 5, simulation results are presented followed by concluding remarks in Section 6.

\section{Preliminary Notations}

Graph plays an important role in representing the communication topology among multiagents. Therefore, some basic notions and results in algebraic graph theory $[36,37]$ and a algebraic inequality [38] are provided.

Let $\mathscr{G}(\mathscr{V}, \mathscr{E}, \mathscr{A})$ be a directed graph of order $n$, where $\mathscr{V}=\left\{v_{1}, \ldots, v_{n}\right\}$ is the set of nodes, $\mathscr{E} \subseteq \mathscr{V} \times \mathscr{V}$ is the set of edges, and $\mathscr{A}=\left[a_{i j}\right]_{n \times n}$ is a weighted adjacency matrix such that $\left(v_{i}, v_{j}\right) \in \mathscr{E}(A) \Leftrightarrow a_{j i}>0$. An edge of $\mathscr{G}(\mathscr{V}, \mathscr{E}, \mathscr{A})$ is denoted by $\varepsilon_{i j}=\left(v_{i}, v_{j}\right)$, where the first element $v_{i}$ and the other element $v_{j}$ are called the root and child vertices, respectively. The set of neighbors of vertex $v_{i}$ is defined as $\mathcal{N}\left(\mathscr{G}(\mathscr{V}, \mathscr{E}, \mathscr{A}), v_{i}\right)=\left\{v_{j}:\left(v_{j}, v_{i}\right) \in \mathscr{E}\right\}$. The corresponding index set is denoted by $\mathscr{N}(\mathscr{G}(\mathscr{V}, \mathscr{E}, \mathscr{A}), i)=\left\{j: v_{j} \epsilon\right.$ $\left.\mathcal{N}\left(\mathscr{G}, v_{i}\right)\right\}$. The Laplacian of the graph $\mathscr{G}(\mathscr{V}, \mathscr{E}, \mathscr{A})$ is defined as $L=\mathscr{D}-\mathscr{A}$, where $\mathscr{D}=\left[d_{i j}\right]_{n \times n}$ is a diagonal matrix with $d_{i i}=\sum_{i=1}^{n} a_{i j}$. A path of graph $\mathscr{G}(\mathscr{V}, \mathscr{E}, \mathscr{A})$ is a sequence $v_{1}, \ldots, v_{k}$ of vertices satisfying $\left(v_{i_{j}}, v_{i_{j+1}}\right) \in \mathscr{E}$. A directed graph is said to be strongly connected, if there exists a directed path between each pair of distinct vertices. A directed tree is a directed graph such that each vertex has only one parent vertex, except one special vertex (root vertex) without any parent vertex.

Directed graph $\mathscr{G}_{s}\left(\mathscr{V}_{s}, \mathscr{E}_{s}, \mathscr{A}_{s}\right)$ is a subgraph of $\mathscr{G}(\mathscr{V}$, $\mathscr{E}, \mathscr{A})$ such that $\mathscr{V}_{s} \subseteq \mathscr{V}, \mathscr{E}_{s} \subseteq \mathscr{E}$. If $\mathscr{V}_{s}=\mathscr{V}, \mathscr{E}_{s} \subseteq$ $\mathscr{E}, \mathscr{G}_{s}\left(\mathscr{V}_{s}, \mathscr{E}_{s}, \mathscr{A}_{s}\right)$ is called a spanning subgraph. We say $\mathscr{G}_{s}\left(\mathscr{V}_{s}, \mathscr{E}_{s}, \mathscr{A}_{s}\right)$ is induced by $\mathscr{V}_{s}$, if, for any $\left(v_{i}, v_{j}\right) \in \mathscr{E}_{s}$, $\left(v_{i}, v_{j}\right) \in \mathscr{E}_{s} \Leftrightarrow\left(v_{i}, v_{j}\right) \in \mathscr{E}$. A spanning tree of $\mathscr{G}(\mathscr{V}$, $\mathscr{E}, \mathscr{A})$ is a directed tree as well as a spanning subgraph of $\mathscr{G}(\mathscr{V}, \mathscr{E}, \mathscr{A})$. A strongly connected component of $\mathscr{G}(\mathscr{V}$, $\mathscr{E}, \mathscr{A})$ is an induced subgraph and is subjected to be strongly connected. Suppose that graph $\mathscr{G}(\mathscr{V}, \mathscr{E}, \mathscr{A})$ has $k$ strongly connected components, denoted by $\mathscr{G}_{1}^{c}\left(\mathscr{V}_{1}^{c}\right.$, $\left.\mathscr{E}_{1}^{c}, \mathscr{A}_{1}^{c}\right), \ldots, \mathscr{G}_{k}^{c}\left(\mathscr{V}_{k}^{c}, \mathscr{E}_{k}^{c}, \mathscr{A}_{k}^{c}\right)$. According to the above definitions, each vertex of directed graph $\mathscr{G}(\mathscr{V}, \mathscr{E}, \mathscr{A})$ lies exactly in one strongly connected component and the strongly connected component of $\mathscr{G}(\mathscr{V}, \mathscr{E}, \mathscr{A})$ partitions its vertices. Another directed graph $\mathscr{G}^{c}\left(\mathscr{V}^{c}, \mathscr{E}^{c}, \mathscr{A}^{c}\right)$ is presented in [37], where $\mathscr{V}^{c}$ consists of all strongly connected components $\mathscr{G}_{1}^{c}\left(\mathscr{V}_{1}^{c}, \mathscr{E}_{1}^{c}, \mathscr{A}_{1}^{c}\right), \ldots, \mathscr{G}_{k}^{c}\left(\mathscr{V}_{k}^{c}, \mathscr{E}_{k}^{c}, \mathscr{A}_{k}^{c}\right)$ of $\mathscr{G}(\mathscr{V}, \mathscr{E}, \mathscr{A})$, and, for any $i, j \in\{1, \ldots, k\},\left(\mathscr{G}_{i}^{c}\left(\mathscr{V}_{i}^{c}, \mathscr{E}_{i}^{c}, \mathscr{A}_{j}^{c}\right), \mathscr{G}_{j}^{c}\left(\mathscr{V}_{j}^{c}, \mathscr{E}_{j}^{c}, \mathscr{A}_{j}^{c}\right)\right) \in \mathscr{E}^{c}$ if only if there exist $v_{m} \in \mathscr{V}_{i}^{c}$ and $v_{n} \in \mathscr{V}_{j}^{c}$ such that $\left(v_{m}, v_{n}\right) \in$ $\mathscr{E}$.

Without causing any confusion, $\mathscr{G}(\mathscr{A})$ can be used to denote the directed graph $\mathscr{G}(\mathscr{V}, \mathscr{E}, \mathscr{A})$.

Definition 1. For directed graph $\mathscr{G}(\mathscr{A})$, if there exist some scalars $\omega_{i}>0, i \in I_{n}$, yields $\omega_{i} a_{i j}=\omega_{j} a_{j i}$, and then it can be said that $\mathscr{G}$ satisfies the detailed balance condition or $\mathscr{G}(\mathscr{A})$ is a detail-balanced graph.

Definition 2. Let $x=\left[x_{1}, \ldots, x_{n}\right] \in \mathbb{R}^{n}$, and then $x$ is called a positive vector if $x_{i}>0, i \in I_{n}$.

Definition 3. For $x \in \mathbb{R}$,

$$
\operatorname{sign}(x)= \begin{cases}1 & x>0 \\ 0 & x=0 \\ -1 & x<0 .\end{cases}
$$

Lemma $4\left(C_{r}-\right.$ inequality [38]). For any $a=\left[a_{1}, \ldots, a_{n}\right] \in$ $\mathbb{R}^{n}, p>0$, and then

$$
\begin{aligned}
\left(\sum_{i=1}^{n}\left|a_{i}\right|\right)^{p} & \leq C \sum_{i=1}^{n}\left|a_{i}\right|^{p}, \\
C & =1, \quad 0<p \leq 1, \\
C & =n^{p-1}, \quad p>1 .
\end{aligned}
$$

Lemma 5 (see [39]). Suppose that there exists a positive definite continuous function $V: \mathscr{D} \rightarrow \mathbb{R}$ such that the following condition holds. 
There exist real numbers $c>0$ and $\rho \in(0,1)$ and an open neighborhood $\mathscr{V} \subseteq \mathscr{D}$ of the origin such that

$$
\dot{V}(x)+c V^{\rho}(x) \leq 0, \quad x \in \mathscr{V} \backslash\{0\} .
$$

Then, the origin is finite-time stable and, depending on the initial sate $x(0)=x_{0}$, the setting time satisfies the following:

$$
T\left(x_{0}\right) \leq \frac{V\left(x_{0}\right)}{c(1-\rho)} .
$$

\section{Problem Formulation}

The multiple mobile agent system studied in this paper consists of $n$ autonomous agents, for example, particles or robots, labeled $1-n$. All these agents share a common state space $\mathbb{R}$. For convenience, $I_{n}$ is used to denote the set $\{1,2, \ldots, n\}$. The state of agent $i$ is denoted by $x_{i}, i \in I_{n}$, and let $x=\left[x_{1}, x_{2}, \ldots, x_{n}\right]^{T}$.

Suppose that the communication topology is $\mathscr{G}(A)$ and the agent $i, i \in I_{n}$, is with the following dynamics:

$$
\dot{x}_{i}(t)=u_{i}(t),
$$

where $u_{i}(t)$ is the protocol to be designed.

The following information exchange protocol is used to solve a weighted-average finite-time consensus problem:

$$
\begin{aligned}
u_{i}(t)= & \alpha \sum_{j \in \mathcal{N}(\mathscr{G}(A), i)} a_{i j} \operatorname{sign}\left(x_{j}-x_{i}\right)\left|x_{j}-x_{i}\right|^{\beta} \\
& +\gamma \sum_{j \in \mathcal{N}(\mathscr{G}(A), i)} a_{i j}\left(x_{j}-x_{i}\right),
\end{aligned}
$$

where $\alpha>0,0<\beta<1, \gamma \geq 0$.

Particularly, the following cases can be obtained:

(i) For $\alpha=0, \gamma=1$, then

$$
u_{i}(t)=\sum_{j \in \mathcal{N}(\mathscr{G}(A), i)} a_{i j}\left(x_{j}-x_{i}\right),
$$

and the nonlinear protocol (7) reduces to the typical linear protocol proposed by Olfati-Saber and Murray [19]. The dynamical behaviors of the multiagents have received considerable attention under this linear protocol and significant results have been established. Further details can be found in the survey papers $[2,18]$.

(ii) $\alpha>0,0<\beta<1, \gamma=0$, and the consensus transforms into the nonlinear protocol proposed by Feng and Long [30]. Feng and Long have proved that their nonlinear protocol can solve the finitetime average-agreement problem and can be applied to the systems with switching topologies assuming that the communication topology is represented by a connected undirected graph.

(iii) $\alpha \neq 0, \beta=0, \gamma=0$, and in this case the protocol becomes discontinuous with respect to the state variables. The research on this topic is still under development. It is worth mentioning that the case when $\alpha \neq 0, \beta=0, \gamma=0$, and the adjacency matrix $A$ of the communication topology $\mathscr{G}(A)$ is a $0-1$ symmetric matrix has been addressed in [40].

\section{Theoretical Analysis}

This section describes some theoretical results of the agents dynamics under the proposed protocol.

4.1. Fixed Communication Topology. In this subsection, dynamic behaviors of the multiagents under fixed topology are considered.

Theorem 6. If the communication topology $\mathscr{G}(A)$ has a spanning tree and each strongly connected component is detailbalanced, then the nonlinear protocol (6) can ensure that the states of agents converge to a weighted-average consensus in finite time.

Proof. The proof is similar to that of [37] and is divided into the following three steps.

Step 1. Suppose that $\mathscr{G}(A)$ is strongly connected and detailbalanced.

As $\mathscr{G}(A)$ is a strongly connected and detail-balanced graph, according to Definition 1 , there exists a positive vector $\omega=\left[\omega_{1}, \omega_{2}, \ldots, \omega_{n}\right]^{T} \in \mathbb{R}^{n}$ such that $\omega_{i} a_{i j}=\omega_{j} a_{j i}$ for all $i, j \in I_{n}$. Then, the following can be obtained:

$$
\sum_{i=1}^{n} \omega_{i} \dot{x}_{i}(t)=0 .
$$

Let

$$
\Delta=\frac{1}{\sum_{i=1}^{n} \omega_{i}} \sum_{i=1}^{n} \omega_{i} x_{i}(t)
$$

Then $\Delta$ is a constant and can be considered as a weightedaverage of the initial sates of the agents. Let $\delta(t)=$ $\left[\delta_{1}(t), \ldots, \delta_{n}(t)\right]^{T}$, where $\delta_{i}(t)=x_{i}(t)-\Delta$. Taking Lyapunov function $V_{1}(t)=(1 / 2) \sum_{i=1}^{n} \omega_{i} \delta_{i}(t)^{2}$, then $\dot{\delta}_{i}(t)=\dot{x}_{i}(t)$ and $\omega^{T} \delta(t)=0$. In [19], $\delta(t)$ is referred to as the group disagreement vector. In the following analysis, $\delta(t)$ is assumed to be a nonzero vector.

Differentiating $V_{1}(t)$ with respect to $t$ provides

$$
\begin{aligned}
& \frac{d V_{1}(t)}{d t}=\sum_{i=1}^{n} \omega_{i} \delta_{i}(t) \dot{\delta}_{i}(t)=\alpha \sum_{i=1}^{n} \omega_{i} \delta_{i}(t) \sum_{j \in \mathcal{N}(\mathscr{G}(A), i)} a_{i j} \\
& \cdot \operatorname{sign}\left(\delta_{j}(t)-\delta_{i}(t)\right)\left|\delta_{j}(t)-\delta_{i}(t)\right|^{\beta}+\gamma \sum_{i=1}^{n} \omega_{i} \delta_{i}(t) \\
& \cdot \sum_{j \in \mathcal{N}(\mathscr{G}(A), i)} a_{i j}\left(\delta_{j}(t)-\delta_{i}(t)\right) \\
& =\frac{1}{2}\left\{\left[\alpha \sum_{i, j=1}^{n} \omega_{i} a_{i j} \delta_{i}(t) \operatorname{sign}\left(\delta_{j}(t)-\delta_{i}(t)\right)\right.\right.
\end{aligned}
$$




$$
\begin{aligned}
& \left.\cdot\left|\delta_{j}(t)-\delta_{i}(t)\right|^{\beta}+\gamma \sum_{i, j=1}^{n} \omega_{i} a_{i j} \delta_{i}(t)\left(\delta_{j}-\delta_{i}\right)\right] \\
& +\left[\alpha \sum_{i, j=1}^{n} \omega_{j} a_{j i} \delta_{j}(t) \operatorname{sign}\left(\delta_{i}(t)-\delta_{j}(t)\right)\right. \\
& \left.\left.\cdot\left|\delta_{i}(t)-\delta_{j}(t)\right|^{\beta}+\gamma \sum_{i, j=1}^{n} \omega_{j} a_{j i} \delta_{j}(t)\left(\delta_{i}-\delta_{j}\right)\right]\right\} \\
& =-\frac{1}{2}\left[\alpha \sum_{i, j=1}^{n} \omega_{i} a_{i j}\left|\delta_{j}-\delta_{i}\right|^{1+\beta}\right. \\
& \left.+\gamma \sum_{i, j=1}^{n} \omega_{i} a_{i j}\left(\delta_{j}-\delta_{i}\right)^{2}\right]^{2}-\frac{\alpha}{2} \sum_{i, j=1}^{n}\left[\left(\omega_{i} a_{i j}\right)^{2 /(1+\beta)}\right. \\
& \left.\cdot\left(\delta_{j}-\delta_{i}\right)^{2}\right]^{(1+\beta) / 2}-\frac{\gamma}{2} \sum_{i, j=1}^{n} \omega_{i} a_{i j}\left(\delta_{j}-\delta_{i}\right)^{2} .
\end{aligned}
$$

According to $C_{r}$-inequality

$$
\begin{aligned}
\frac{d V_{1}(t)}{d t} \leq & -\frac{\alpha}{2}\left[\sum_{i, j=1}^{n}\left(\omega_{i} a_{i j}\right)^{2 /(1+\beta)}\left(\delta_{j}-\delta_{i}\right)^{2}\right]^{(1+\beta) / 2} \\
& -\frac{\gamma}{2} \sum_{i, j=1}^{n} \omega_{i} a_{i j}\left(\delta_{j}-\delta_{i}\right)^{2} .
\end{aligned}
$$

Let $M_{1}(\delta(t))=\sum_{i, j=1}^{n}\left(\omega_{i} a_{i j}\right)^{2 /(1+\beta)}\left(\delta_{j}-\delta_{i}\right)^{2}, N_{1}(\delta(t))=$ $\sum_{i, j=1}^{n} \omega_{i} a_{i j}\left(\delta_{j}-\delta_{i}\right)^{2}$ and $B=\left[b_{i j}\right]_{n \times n}, C=\left[c_{i j}\right]_{n \times n}$, where $b_{i j}=\left(\omega_{i} a_{i j}\right)^{2 /(1+\beta)}, c_{i j}=\omega_{i} a_{i j}$. It is obvious that $B=B^{T}$, $C=C^{T}, L(B)$, and $L(C)$ denote the Laplacian matrices of the graphs $\mathscr{G}(B)$, and $\mathscr{G}(C)$, respectively. Let $\lambda(L(B))=$ $\left[\lambda_{1}, \lambda_{2}, \ldots, \lambda_{n}\right]$, where $0=\lambda_{1} \leq \lambda_{2} \leq \cdots \leq \lambda_{n}$, represents the spectrum of matrix $L(B)$. It can easily be checked that 0 is an algebraically simple eigenvalue and $\mathbf{1}=[1, \ldots, 1]^{T}$ is the associated eigenvector. As $L(B)$ is a real symmetric matrix, there exists orthogonal matrix $\Phi=\left[\varphi_{1}, \varphi_{2}, \ldots, \varphi_{n}\right]^{T}$, such that $\varphi_{1}=(1 / \sqrt{n})[1,1, \ldots, 1]^{T}$ and $\Phi L(B) \Phi^{T}=\operatorname{diag}(\lambda(B))$. Then

$$
\begin{aligned}
M(\delta(t)) & =2 \delta(t)^{T} L(B) \delta(t) \\
& =2(\Phi \delta(t))^{T} \operatorname{diag}(\lambda(L(B))) \Phi \delta(t)
\end{aligned}
$$

Let $\widetilde{\delta}(t)=\Phi \delta(t)=\left[\widetilde{\delta}_{1}, \widetilde{\delta}_{2}, \ldots, \widetilde{\delta}_{n}\right]^{T}$, and then $\widetilde{\delta}_{1}(t)=$ $(1 / \sqrt{n}) \sum_{i=1}^{n} \delta_{i}(t)$. It is obvious that $\widetilde{\delta}^{T}(t) \widetilde{\delta}(t)=\delta^{T}(t) \delta(t)$. According the above analysis, (12) can be rewritten as

$$
\begin{aligned}
& M(\delta(t))=2\left[\lambda_{2}(L(B)) \widetilde{\delta}_{2}(t)^{2}+\lambda_{3}(L(B)) \widetilde{\delta}_{3}(t)^{2}\right. \\
& \left.+\cdots+\lambda_{n}(L(B)) \widetilde{\delta}_{n}(t)^{2}\right]
\end{aligned}
$$

$$
\begin{aligned}
& \geq 2\left[\lambda_{2}(L(B)) \tilde{\delta}(t)^{T} \tilde{\delta}(t)\right. \\
& \left.-\frac{\lambda_{2}(L(B))}{n}\left(\sum_{i=1}^{n} \delta_{i}(t)\right)^{2}\right] \\
& =2\left\{\lambda_{2}(L(B)) \tilde{\delta}(t)^{T} \tilde{\delta}(t)\left[1-\frac{\left(\sum_{i=1}^{n} \delta_{i}(t)\right)^{2}}{n \sum_{i=1}^{n} \delta_{i}^{2}(t)}\right]\right\} .
\end{aligned}
$$

Recalling the fact $\omega^{T} \delta(t)=0$, it can be found that $\delta_{1}(t), \delta_{2}(t), \ldots, \delta_{n}(t)$ do not have the same sign. By using the $C_{r}$-inequality, one can obtain

$$
1-\frac{\left(\sum_{i=1}^{n} \delta_{i}(t)\right)^{2}}{n \sum_{i=1}^{n} \delta_{i}^{2}(t)}>1-\frac{\left(\sum_{i=1}^{n}\left|\delta_{i}(t)\right|\right)^{2}}{n \sum_{i=1}^{n} \delta_{i}^{2}(t)} \geq 0 .
$$

Similar to [31], let $\mathscr{U}=\{\xi$ : nonzero elements of $\xi_{1}, \ldots, \xi_{n}$ are not with the same sign $\}$, and let $\mathcal{U}^{0}=\mathcal{U} \bigcap\{\xi$ : $\left.\xi^{T} \xi=1\right\}$. It is obvious that $\mathscr{U}^{0}$ is compact set. For convenience, construct function

$$
W(\delta)=\frac{\left(\sum_{i=1}^{n}\left|\delta_{i}(t)\right|\right)^{2}-\left(\sum_{i=1}^{n} \delta_{i}(t)\right)^{2}}{n \sum_{i=1}^{n} \delta_{i}^{2}(t)} .
$$

Clearly, $W(\delta)>0$. The expression can be rewritten as

$$
W(\delta)=\frac{1}{n}\left[\sum_{i=1}^{n} \frac{\operatorname{sign}\left(\delta_{i}\right) \delta_{i}}{\|\delta\|}-\left(\sum_{i=1}^{n} \frac{\delta_{i}}{\|\delta\|}\right)^{2}\right] .
$$

Let $S(x)=\sum_{i=1}^{n} \operatorname{sign}\left(x_{i}\right) x_{i}$ and $T(x)=\left(\sum_{i=1}^{n} x_{i}\right)^{2}$, where $x=\left[x_{1}, \ldots, x_{n}\right] \in \mathbb{R}^{n}$. Obviously, $S(x)$ and $T(x)$ are continuous functions with respect to $x$. Then, one can get

$$
W(\delta)=W(\zeta)=\frac{1}{n}[S(\zeta)-T(\zeta)] \geq K_{1}>0
$$

where $\zeta=\left[\delta_{1} /\|\delta\|, \ldots, \delta_{n} /\|\delta\|\right]$ and $K_{1}=\min _{\theta \in \mathscr{U}^{0}}(1 /$ $n)[S(\zeta)-T(\zeta)]$. Hence,

$$
\begin{aligned}
M(\delta(t)) & \geq 2 K_{1} \lambda_{2}(L(B)) \delta^{T}(t) \delta(t) \\
& \geq \frac{2 K_{1} \lambda_{2}(L(B))}{\omega_{\max }} \sum_{i=1}^{n} \omega_{i} \delta_{i}(t)^{2} .
\end{aligned}
$$

Similar to the above analysis, there exists a constant $K_{2}>$ 0 such that

$$
\begin{aligned}
N(\delta(t)) & =2 \delta(t)^{T} L(C) \delta(t) \\
& \geq \frac{2 K_{2} \lambda_{2}(L(C))}{\omega_{\max }} \sum_{i=1}^{n} \omega_{i} \delta_{i}(t)^{2}
\end{aligned}
$$

where $\lambda_{2}(L(B)), \lambda_{2}(L(C))$ represents the second largest eigenvalue of the matrixes $L(B)$ and $L(C)$, respectively. 
Let

$$
\begin{aligned}
& K_{3}=\frac{\alpha}{2}\left[\frac{2 K_{1} \lambda_{2}(L(B))}{\omega_{\max }}\right]^{(1+\beta) / 2}, \\
& K_{4}=\frac{4 \gamma K_{2} \lambda_{2}(L(C))}{\omega_{\max }} .
\end{aligned}
$$

Obviously, $K_{3}, K_{4}>0$ and

$$
\frac{d V_{1}(t)}{d t} \leq-\left(K_{3}+K_{4} V_{1}(t)^{2 /(1+\beta)}\right) V_{1}(t)^{(1+\beta) / 2}
$$

The above analysis indicates that protocol (7) is able to solve the weighted-average consensus problem if the interaction topology is represented by a strongly connected detailbalanced graph. The last inequality also indicates that the proposed protocol in this paper is capable of providing a faster convergence rate than the typical linear protocol proposed in [19] and the nonlinear finite-time consensus protocol

$$
u_{i}(t)=\alpha \sum_{j \in \mathcal{N}(\mathscr{G}(A), i)} a_{i j} \operatorname{sign}\left(x_{j}-x_{i}\right)\left|x_{j}-x_{i}\right|^{\beta},
$$

proposed by Feng and Long in [30].

Step 2. Suppose that $\mathscr{G}(A)$ has a spanning tree and the associated root vertex is $v_{i}$ and $a_{i j}=0$, for any $j \in I_{n}$. Furthermore, assume that the subgraph induced by the child vertices $\left\{v_{j}\right\}_{j \in I_{n}, j \neq i}$ is strongly connected and detail-balanced.

Without loss of generality, assume that the root vertex $v_{i}$ is $v_{1}$. Therefore, one can obtain that $a_{11}=a_{12}=\cdots=a_{1 n}=$ 0 and $a_{11}, a_{21}, \ldots, a_{(n-1) 1}$ are not all zeros. $\mathscr{G}(\widetilde{A})$ denotes the subgraph induced by the vertices excluding the vertex $v_{1}$. In this case, as $u_{1}(t) \equiv 0$, the dynamic behaviors of the other agents may be focused. Then, one can obtain

$$
\begin{aligned}
u_{i}(t)= & \alpha \sum_{j \in \mathcal{N}(\mathscr{G}(A), i) \backslash\{1\}} a_{i j} \operatorname{sign}\left(x_{j}-x_{i}\right)\left|x_{j}-x_{i}\right|^{\beta} \\
& +\gamma \sum_{j \in \mathcal{N}(\mathscr{G}(A), i) \backslash\{1\}} a_{i j}\left(x_{j}-x_{i}\right), \quad i \neq 1 .
\end{aligned}
$$

That is,

$$
\begin{aligned}
u_{i}(t)= & \alpha \sum_{j=2}^{n} a_{i j} \operatorname{sign}\left(x_{j}-x_{i}\right)\left|x_{j}-x_{i}\right|^{\beta} \\
& +\gamma \sum_{j=2}^{n} a_{i j}\left(x_{j}-x_{i}\right)+\alpha \\
& \cdot a_{i 1} \operatorname{sign}\left(x_{1}-x_{i}\right)\left|x_{1}-x_{i}\right|^{\beta}+\gamma \\
& \cdot a_{i 1}\left(x_{1}-x_{i}\right), \quad i \neq 1 .
\end{aligned}
$$

Let $\delta_{i}=x_{i}-x_{1}, i \in I_{n}$. It is obvious that $\delta_{1} \equiv 0$. Recalling that $\dot{x}_{1} \equiv 0$, one can get

$$
\begin{aligned}
\dot{\delta}_{i}= & \alpha \sum_{j=2}^{n} a_{i j} \operatorname{sign}\left(\delta_{j}-\delta_{i}\right)\left|\delta_{j}-\delta_{i}\right|^{\beta}+\gamma \sum_{j=2}^{n} a_{i j}\left(\delta_{j}-\delta_{i}\right) \\
& -\alpha a_{i 1} \operatorname{sign}\left(\delta_{i}\right)\left|\delta_{i}\right|^{\beta}-\gamma a_{i 1} \delta_{i}, \quad i \neq 1 .
\end{aligned}
$$

Take the Lyapunov function $V_{2}(t)=(1 / 2) \sum_{i=1}^{n} \omega_{i} \delta_{i}(t)^{2}$. Differentiating $V_{2}(t)$ with respect to $t$ leads to

$$
\begin{aligned}
& \frac{d V_{2}(t)}{d t}=\sum_{i=1}^{n} \omega_{i} \delta_{i}(t) \dot{\delta}_{i}(t)=\sum_{i=2}^{n} \omega_{i} \delta_{i}(t)\left[\alpha \sum_{j=2}^{n} a_{i j}\right. \\
& \cdot \operatorname{sign}\left(\delta_{j}-\delta_{i}\right)\left|\delta_{j}-\delta_{i}\right|^{\beta}+\gamma \sum_{j=2}^{n} a_{i j}\left(\delta_{j}-\delta_{i}\right)-\alpha \\
& \left.\cdot a_{i 1} \operatorname{sign}\left(\delta_{i}\right)\left|\delta_{i}\right|^{\beta}-\gamma \cdot a_{i 1} \delta_{i}\right] \\
& =\frac{1}{2}\left\{\left[\alpha \sum_{i, j=2}^{n} \omega_{i} a_{i j} \delta_{i}(t) \operatorname{sign}\left(\delta_{j}(t)-\delta_{i}(t)\right)\right.\right. \\
& \left.\cdot\left|\delta_{j}(t)-\delta_{i}(t)\right|^{\beta}+\gamma \sum_{i, j=2}^{n} \omega_{i} a_{i j} \delta_{i}(t)\left(\delta_{j}-\delta_{i}\right)\right] \\
& +\left[\alpha \sum_{i, j=2}^{n} \omega_{j} a_{j i} \delta_{j}(t) \operatorname{sign}\left(\delta_{i}(t)-\delta_{j}(t)\right)\right. \\
& \left.\left.\cdot\left|\delta_{i}(t)-\delta_{j}(t)\right|^{\beta}+\gamma \sum_{i, j=2}^{n} \omega_{j} a_{j i} \delta_{j}(t)\left(\delta_{i}-\delta_{j}\right)\right]\right\} \\
& -\alpha \sum_{i=2}^{n} \omega_{i} a_{i 1}\left|\delta_{i}(t)\right|^{1+\beta}-\gamma \sum_{i=2}^{n} \omega_{i} a_{i 1}\left(\delta_{i}(t)\right)^{2} \\
& =-\frac{1}{2}\left[\alpha \sum_{i, j=2}^{n} \omega_{i} a_{i j}\left|\delta_{j}-\delta_{i}\right|^{1+\beta}\right. \\
& \left.+\gamma \sum_{i, j=2}^{n} \omega_{i} a_{i j}\left(\delta_{j}-\delta_{i}\right)^{2}\right]-\alpha \sum_{i=2}^{n}\left[\left(\omega_{i} a_{i 1}\right)^{2 /(1+\beta)}\right. \\
& \left.\cdot\left(\delta_{i}(t)\right)^{2}\right]^{(1+\beta) / 2}-\gamma \sum_{i=2}^{n} \omega_{i} a_{i 1}\left(\delta_{i}(t)\right)^{2} \\
& =-\frac{\alpha}{2}\left\{\sum_{i, j=2}^{n}\left[\left(\omega_{i} a_{i j}\right)^{2 /(1+\beta)}\left(\delta_{j}-\delta_{i}\right)^{2}\right]^{(1+\beta) / 2}\right. \\
& \left.+2 \sum_{i=2}^{n}\left[\left(\omega_{i} a_{i 1}\right)^{2 /(1+\beta)}\left(\delta_{i}(t)\right)^{2}\right]^{(1+\beta) / 2}\right\} \\
& -\frac{\gamma}{2}\left[\sum_{i, j=2}^{n} \omega_{i} a_{i j}\left(\delta_{j}-\delta_{i}\right)^{2}+2 \sum_{i=2}^{n} \omega_{i} a_{i 1}\left(\delta_{i}(t)\right)^{2}\right] .
\end{aligned}
$$


Applying the $C_{r}$-inequality to the above equation, the following is obtained:

$$
\begin{aligned}
& \frac{d V_{2}(t)}{d t} \leq-\frac{\alpha}{2}\left[\sum_{i, j=2}^{n}\left(\omega_{i} a_{i j}\right)^{2 /(1+\beta)}\left(\delta_{j}-\delta_{i}\right)^{2}\right. \\
& \left.+2 \sum_{i=2}^{n}\left(\omega_{i} a_{i 1}\right)^{2 /(1+\beta)}\left(\delta_{i}(t)\right)^{2}\right]^{(1+\beta) / 2} \\
& -\frac{\gamma}{2}\left[\sum_{i, j=2}^{n} \omega_{i} a_{i j}\left(\delta_{j}-\delta_{i}\right)^{2}+2 \sum_{i=2}^{n} \omega_{i} a_{i 1}\left(\delta_{i}(t)\right)^{2}\right] .
\end{aligned}
$$

For simplicity, let

$$
\begin{aligned}
M_{2}(\delta(t))= & \sum_{i, j=2}^{n}\left(\omega_{i} a_{i j}\right)^{2 /(1+\beta)}\left(\delta_{j}-\delta_{i}\right)^{2} \\
& +2 \sum_{i=2}^{n}\left(\omega_{i} a_{i 1}\right)^{2 /(1+\beta)}\left(\delta_{i}(t)\right)^{2}, \\
N_{2}(\delta(t))= & \sum_{i, j=2}^{n} \omega_{i} a_{i j}\left(\delta_{j}-\delta_{i}\right)^{2}+2 \sum_{i=2}^{n} \omega_{i} a_{i 1}\left(\delta_{i}(t)\right)^{2} .
\end{aligned}
$$

Let $\widetilde{A}=\left[\widetilde{a}_{i j}\right]_{(n-1) \times(n-1)}$, where $\widetilde{a}_{i j}=\left(\omega_{i+1} a_{(i+1)(j+1)}\right)^{2 /(1+\beta)}$ denoted $\widetilde{b}=\left[\left(\omega_{2} a_{21}\right)^{2 /(1+\beta)}, \ldots,\left(\omega_{n} a_{n 1}\right)^{2 /(1+\beta)}\right]$. Then one can get that

$$
M_{2}(\delta(t))=2 \delta^{T} L(\widetilde{A}) \delta(t)+2 \delta^{T} \operatorname{diag}(\widetilde{b}) \delta
$$

Then, $\widetilde{B}=L(\widetilde{A})+\operatorname{diag}(\widetilde{b})$ is real symmetric and positive definite. Let the smallest eigenvalue of $\widetilde{B}$ be $\lambda_{1}(\widetilde{B})$. Then, $\lambda_{1}(\widetilde{B})>$ 0 , and, for any nonzero vector $\eta \in \mathbb{R}^{n-1}, \eta^{T} \widetilde{B} \eta \geq \lambda_{1}(\widetilde{B}) \eta^{T} \eta$. Therefore,

$$
M_{2}(\delta(t)) \geq 2 \lambda_{1}(\widetilde{B}) \delta^{T} \delta \geq K_{5} \sum_{i=1}^{n} \omega_{i} \delta_{i}^{2}
$$

where $K_{5}=2 \lambda_{1}(\widetilde{B}) / \omega_{\max }$.

Similarly, one can obtain that

$$
N_{2}(\delta(t)) \geq K_{6} \sum_{i=1}^{n} \omega_{i} \delta_{i}^{2}
$$

Then, the following inequality holds:

$$
\begin{aligned}
& \frac{d V_{2}(t)}{d t} \\
& \quad \leq-\left[\frac{\alpha}{2} K_{5}^{(1+\beta) / 2}+\frac{\gamma}{2} K_{6} V_{2}^{2 /(1+\beta)}(t)\right] V_{2}^{(1+\beta) / 2}(t) .
\end{aligned}
$$

The above analysis indicates that protocol (7) is able to solve the weighted-average consensus problem if $\mathscr{G}(A)$ has a spanning tree and each strongly connected component is detail-balanced.
Step 3. Suppose that $\mathscr{G}(A)$ has a spanning tree and each strongly connected component is detail-balanced. Then, the proposed protocol will solve a finite-time weighted-average consensus problem.

Consider the directed graph $\mathscr{G}^{c}(A)$ consisting of the strongly connected components of $\mathscr{G}(A)$. Obviously, $\mathscr{G}^{c}(A)$ is a directed tree. From the definition of $\mathscr{G}^{c}(A)$, the following facts can be obtained:

(i) The dynamic behaviors of agents belonging to the vertex set that corresponds to the root vertex of $\mathscr{G}^{c}(A)$ are not affected by others and the local interaction topology among them is strongly connected and detail-balanced. Their states will reach an agreement in a finite time in accordance with the conclusion of Step 1. Considering $\mathscr{G}^{c}(A)$, its root vertex (corresponding to nonempty subset of $\mathscr{V}$, where $\mathscr{V}$ is the vertices set of graph $\mathscr{G}(A))$ is labeled 1 and the final consensus state is denoted by $x_{0}$.

(ii) Consider the dynamics of agents, denoted by $v_{i_{1}}, \ldots, v_{i_{k_{i}}}$, corresponding to vertex $i$ (not the root vertex) of $\mathscr{G}^{c}(A)$. Their dynamic behaviors are only affected by such agents, where there exists at least one vertex that belongs to set $\left\{v_{l}, l=1, \ldots, k_{i}\right\}$ and they can provide information; that is, there exist directed paths connecting them to $v_{l}, l=1, \ldots, k_{i}$. Suppose such agents excluding $v_{l}, l=1, \ldots, k_{i}$, are $v_{j_{1}}, v_{j_{2}}, \ldots, v_{j_{m_{i}}}$, their sates have already reached consensus, and the consensus state is $x_{0}$. Then, for any $l \in\left\{1,2, \ldots, k_{i}\right\}$,

$$
\begin{aligned}
& \sum_{s=1}^{k_{i}} a_{i_{l}, i_{s}}\left(x_{i_{s}}-x_{i_{l}}\right)+\sum_{s=1}^{m_{i}} a_{i_{l}, j_{s}}\left(x_{j_{s}}-x_{i_{l}}\right) \\
& \quad=\sum_{s=1}^{k_{i}} a_{i_{l}, i_{s}}\left(x_{i_{s}}-x_{i_{l}}\right)+\left(\sum_{s=1}^{m_{i}} a_{i_{l}, j_{s}}\right)\left(x_{0}-x_{i_{l}}\right) .
\end{aligned}
$$

Therefore, $v_{j_{1}}, \ldots, v_{j_{k_{i}}}$ can be seen as one (virtual) agent and as the leader of $v_{i_{l}}, l=1, \ldots, k_{i}$. If one relabels the vertices $v_{i_{l}}, l=1, \ldots, k_{i}$, by $v_{l}, l=$ $2, \ldots, k_{i}+1$, and labels the (virtual) agent by 1 , then the communication topology of agents $v_{l}, l=1, \ldots, k_{i}+$ 1 , is $G\left(A^{0}\right)$, where

$$
A^{0}=\left(\begin{array}{cccc}
0 & 0 & \cdots & 0 \\
\sum_{s=1}^{m_{i}} a_{i_{1}, j_{s}} & a_{i_{1}, i_{2}} & \cdots & a_{i_{1}, i_{k_{i}}} \\
\vdots & \vdots & \vdots & \vdots \\
\sum_{s=1}^{m_{i}} a_{i_{k_{i}}, j_{s}} & a_{i_{k_{i}}, i_{2}} & \cdots & a_{i_{k_{i}}, i_{k_{i}}}
\end{array}\right) .
$$

Recalling the theoretical results produced in Step 2, the multiagents $v_{l}, l=1, \ldots, k_{i}+1$, will reach 
consensus in finite time, and the consensus state will be $x_{0}$.

(iii) In accordance with the above presented conclusion, the proposed protocol has solved a finite-time weighted-average consensus problem, and the consensus state is $x_{0}$.

The proof is completed.

4.2. Switching Topology. In practice, the information channel among agents may not always be available due to the physical equipment restrictions or the external interferences in the signal, such as exceeding the sensing range or existence of obstacles between the agents. Therefore, it would be reasonable to assume that the communication topology is dynamically changing.

Theorem 7. Suppose that $\mathscr{G}(A(t))$ is strongly connected and detail-balanced for all $t \geq 0$. Then, protocol (7) will solve a finite-time weighted-average consensus under time-varying topology $\mathscr{G}(A(t))$ if there exists a common positive vector $\omega=$ $\left[\omega_{1}, \omega_{2}, \ldots, \omega_{n}\right]^{T} \in \mathbb{R}^{n}$ such that $\omega_{i} a_{i j}(t)=\omega_{j} a_{j i}(t)$ for all $i, j \in I_{n}$.

Proof. The proof is similar to that of Theorem 6 with the same notations, such as $\Delta$ and $\delta(t)$. Consider the Lyapunov function $V_{3}(\delta(t))$, then the constants, such as $K_{3}, K_{4}, K_{5}$, $K_{6}$ that are derived in proof of Theorem 6, will transform into time-varying parameters $K_{3}(t), K_{4}(t), K_{5}(t), K_{6}(t)$. Take $K_{i}(t)=\min _{i} K_{i}(t), i \in 3,4,5,6$, and then the multiagents will reach an agreement in finite time.

It should be noted that a common Lyapunov can be constructed for the consensus error system if $\mathscr{G}(A(t))$ is strongly connected and detail-balanced for all $t \geq 0$. Based on this condition, a preliminary result for finite-time consensus of multiagent system is given in theorem. However, how to reach finite-time consensus in multiagent system with switching topology without the condition that $\mathscr{G}(A(t))$ is a strongly connected and detail-balanced for all $t \geq 0$ is still an unsolved issue.

\section{Simulation and Applications}

In this section, some simulations are performed by fixing $\alpha=$ $4, \beta=0.5$, and $\gamma=3$ in order to validate the effectiveness of the theoretical results.

Two digraphs with $n=6$ and $n=7$ are presented in Figures 1 and 2, respectively. The adjacency matrix of $\mathscr{G}_{1}$ is

$$
A=\left(\begin{array}{cccccc}
0 & 3 & 0 & 0 & 0 & 2 \\
2 & 0 & 3 & 0 & 0 & 0 \\
0 & 9 & 0 & 3 & 0 & 0 \\
0 & 0 & 0.75 & 0 & 5 & 0 \\
0 & 0 & 0 & 10 & 0 & 3 \\
0.8 & 0 & 0 & 0 & 1.2 & 0
\end{array}\right) .
$$

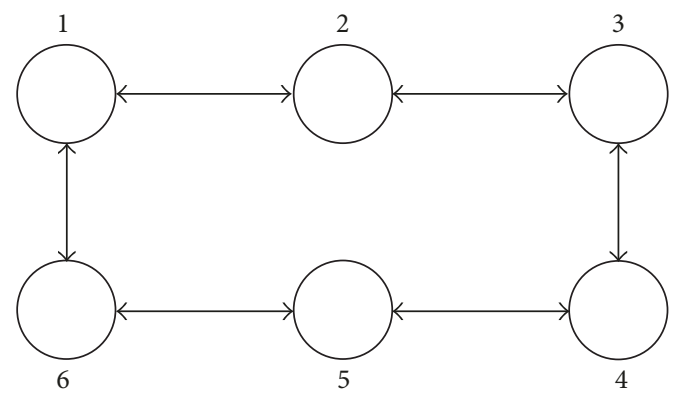

FIGURE 1: Digraph $\mathscr{G}_{1}$.

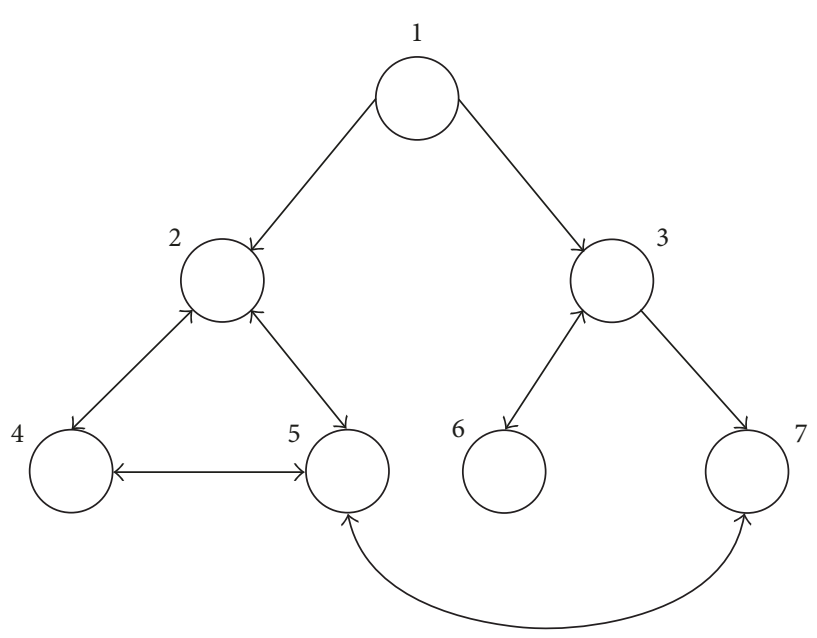

FIGURE 2: Digraph $\mathscr{G}_{2}$.

It can be seen that $\mathscr{G}_{1}$ is detailed balanced graph and $\omega_{1}=[2,3,1,4,2,5]^{T}$ is satisfying $\omega_{i} a_{i j}=\omega_{j} a_{j i}$. The adjacency matrix of $\mathscr{G}_{2}$ is

$$
A=\left(\begin{array}{ccccccc}
0 & 0 & 0 & 0 & 0 & 0 & 0 \\
3 & 0 & 0 & 3 & 4 & 0 & 0 \\
6 & 0 & 0 & 0 & 0 & 0 & 0 \\
0 & 0.6 & 0 & 0 & 2 & 0 & 10 \\
0 & 1 & 0 & 2.5 & 0 & 0 & 25 \\
0 & 0 & 7 & 0 & 0 & 0 & 0 \\
0 & 0 & 0 & 0 & 50 & 0 & 0
\end{array}\right)
$$

Digraph $\mathscr{G}_{2}$ has a spanning tree and each strongly connected component is detail-balanced. And $\omega_{2}=$ $[2,1,3,5,4,6,2]^{T}$ is satisfying $\omega_{i} a_{i j}=\omega_{j} a_{j i}$.

Figures 3 and 4 provide the dynamic behaviors of the multiagents under the interaction topology $\mathscr{G}_{1}$ with initial state $x(0)=[3,4,7,-6,8,5]$ and the state trajectories of agents for communication $\mathscr{G}_{2}$ with initial state $x(0)=[3,-4,7,-6,2,5,6]$, respectively. The simulation results demonstrate that the multiagents can reach a finitetime consensus under the proposed protocol. 


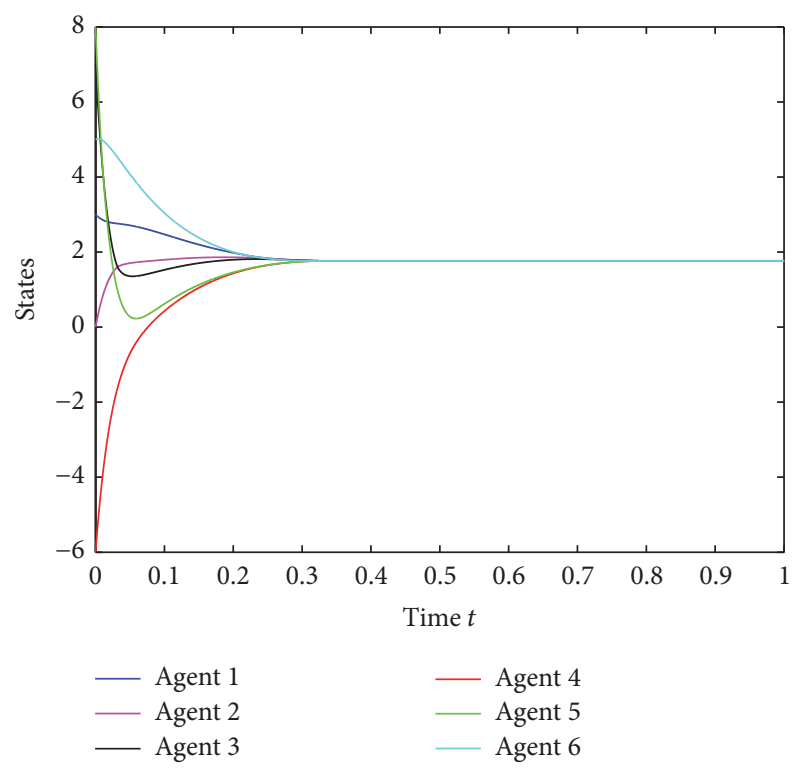

FIGURE 3: Dynamics of agents under the communication topology $\mathscr{G}_{2}$.

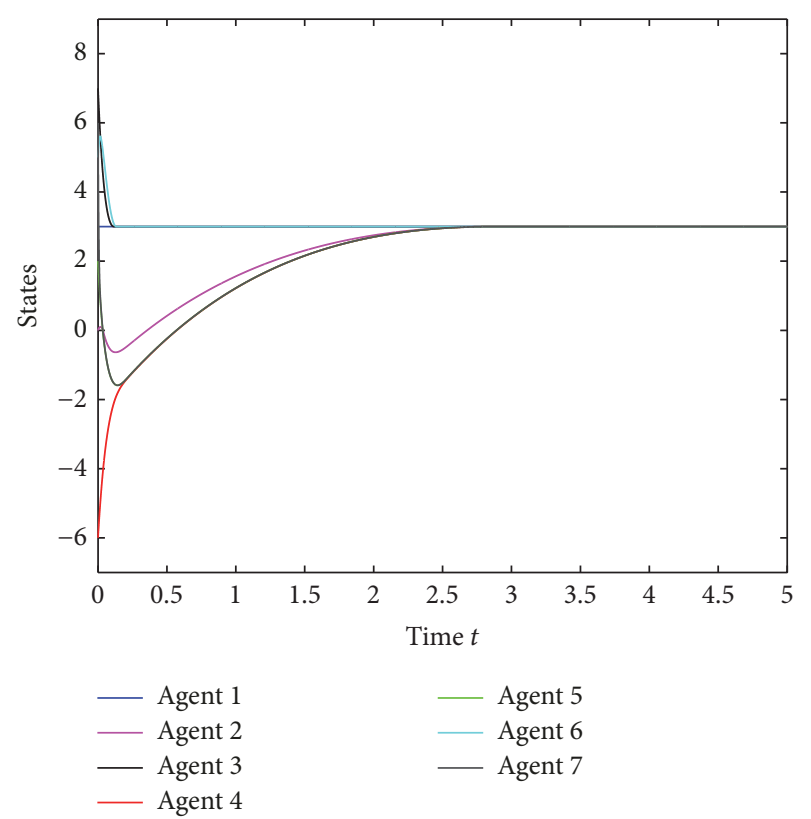

Figure 4: Dynamics of agents under the communication topology $\mathscr{G}_{2}$.

\section{Conclusion}

In this paper, finite-time consensus problem in multiagent networks with first-order dynamics has been investigated and examined. A new nonlinear information exchange protocol has been developed to ensure the achievement of consensus. The designed protocol ensures that the states of the agents converge to a weighted-average consensus in a finite time, provided that the communication topology is a weighted directed graph with a spanning tree and each strongly connected component is detail-balanced. Furthermore, the theoretical analysis has demonstrated that the proposed protocol is also capable of solving the finite-time consensus problem of networks with a switching topology. Future work will focus on achieving finite-time consensus with nonlinear intrinsic dynamics and time-varying topology.

\section{Conflicts of Interest}

The authors declare that they have no conflicts of interest.

\section{References}

[1] R. W. Beard, T. W. McLain, M. A. Goodrich, and E. P. Anderson, "Coordinated target assignment and intercept for unmanned air vehicles," IEEE Transactions on Robotics and Automation, vol. 18, no. 6, pp. 911-922, 2002.

[2] W. Ren, R. W. Beard, and E. M. Atkins, "Information consensus in multivehicle cooperative control," IEEE Control Systems Magazine, vol. 27, no. 2, pp. 71-82, 2007.

[3] G. Wen, Z. Duan, G. Chen, and W. Yu, "Consensus tracking of multi-agent systems with Lipschitz-type node dynamics and switching topologies," IEEE Transactions on Circuits and Systems I. Regular Papers, vol. 61, no. 2, pp. 499-511, 2014.

[4] T. Vicsek, A. Czirók, E. Ben-Jacob, I. Cohen, and O. Shochet, "Novel type of phase transition in a system of self-driven particles," Physical Review Letters, vol. 75, no. 6, pp. 1226-1229, 1995.

[5] A. Jadbabaie, J. Lin, and A. S. Morse, "Coordination of groups of mobile autonomous agents using nearest neighbor rules," IEEE Transactions on Automatic Control, vol. 48, no. 6, pp. 988-1001, 2003.

[6] J. Toner and Y. Tu, "Long-range order in a two-dimensional dynamical XY model: how birds fly together," Physical Review Letters, vol. 75, no. 23, pp. 4326-4629, 1995.

[7] G. Wen, Z. Duan, G. Chen, and X. Geng, "A weighted localworld evolving network model with aging nodes," Physica A: Statistical Mechanics and Its Applications, vol. 390, no. 21-22, pp. 4012-4026, 2011.

[8] L. Wang, H. Shi, T. Chu, W. Zhang, and L. Zhang, "Aggregation of forging swarms," in Lecture Notes in Artificial Intelligence, vol. 3339, pp. 766-777, Springer-Verlag, 2004.

[9] C. Li, X. Yu, Z. Liu, and T. Huang, "Asynchronous impulsive containment control in switched multi-agent systems," Information Sciences, vol. 370-371, pp. 667-679, 2016.

[10] G. Wen, W. Yu, X. Yu, and Lü J., "Complex cyber-physical networks: from cybersecurity to security control," Journal of Systems Science \& Complexity, vol. 30, no. 1, pp. 46-67, 2017.

[11] G. Chen, Z. Y. Dong, D. J. Hill, and Y. S. Xue, "Exploring reliable strategies for defending power systems against targeted attacks," IEEE Transactions on Power Systems, vol. 26, no. 3, pp. 10001009, 2011.

[12] G. Chen, Z. Y. Dong, D. J. Hill, and G. H. Zhang, "An improved model for structural vulnerability analysis of power networks," Physica A: Statistical Mechanics and Its Applications, vol. 388, no. 19, pp. 4259-4266, 2009.

[13] G. Wen, G. Hu, J. Hu, X. Shi, and G. Chen, "Frequency regulation of source-grid-load systems: a compound control strategy," IEEE Transactions on Industrial Informatics, vol. 12, no. 1, pp. 69-78, 2016. 
[14] C. Li, X. Yu, W. Yu, T. Huang, and Z. Liu, "Distributed eventtriggered scheme for economic dispatch in smart grids," IEEE Transactions on Industrial Informatics, vol. 12, no. 5, pp. 17751785, 2016.

[15] H. Li, G. Chen, X. Liao, and T. Huang, "Attraction region seeking for power grids," IEEE Transactions on Circuits and Systems II: Express Briefs, vol. 64, no. 2, pp. 201-205, 2017.

[16] G. Chen, C. Li, and Z. Y. Dong, "Parallel and distributed computation for dynamical economic dispatch," IEEE Transactions on Smart Grid, vol. 8, no. 2, pp. 1026-1027, 2017.

[17] C. Li, X. Yu, W. Yu, G. Chen, and J. Wang, "Efficient computation for sparse load shifting in semand side management," IEEE Transactions on Smart Grid, vol. 8, no. 1, pp. 250-261, 2017.

[18] R. Olfati-Saber, J. A. Fax, and R. M. Murray, "Consensus and cooperation in networked multi-agent systems," Proceedings of the IEEE, vol. 95, no. 1, pp. 215-233, 2007.

[19] R. Olfati-Saber and R. M. Murray, "Consensus problems in networks of agents with switching topology and time-delays," IEEE Transactions on Automatic Control, vol. 49, no. 9, pp. 15201533, 2004.

[20] W. Ren and R. W. Beard, "Consensus seeking in multiagent systems under dynamically changing interaction topologies," IEEE Transactions on Automatic Control, vol. 50, no. 5, pp. 655661, 2005.

[21] Z. Lin, M. Broucke, and B. Francis, "Local control strategies for groups of mobile autonomous agents," IEEE Transactions on Automatic Control, vol. 49, no. 4, pp. 622-629, 2004.

[22] Z. Lin, B. Francis, and M. Maggiore, "Necessary and sufficient graphical conditions for formation control of unicycles," IEEE Transactions on Automatic Control, vol. 50, no. 1, pp. 121-127, 2005.

[23] M. Porfiri and D. J. Stilwell, "Consensus seeking over random weighted directed graphs," IEEE Transactions on Automatic Control, vol. 52, no. 9, pp. 1767-1773, 2007.

[24] G. Wen, W. Yu, G. Hu, J. Cao, and X. Yu, "Pinning synchronization of directed networks with switching topologies: a multiple Lyapunov functions approach," IEEE Transactions on Neural Networks and Learning Systems, vol. 26, no. 12, pp. 3239-3250, 2015.

[25] F. Xiao and L. Wang, "Asynchronous consensus in continuoustime multi-agent systems with switching topology and timevarying delays," IEEE Transactions on Automatic Control, vol. 53, no. 8, pp. 1804-1816, 2008.

[26] L. Fang and P. J. Antsaklis, "Asynchronous consensus protocols using nonlinear paracontractions theory," IEEE Transactions on Automatic Control, vol. 53, no. 10, pp. 2351-2355, 2008.

[27] F. Xiao and L. Wang, "Consensus problems for high-dimensional multi-agent systems," IET Control Theory and Applications, vol. 1, no. 3, pp. 830-837, 2007.

[28] G. Wen, Z. Duan, W. Yu, and G. Chen, "Consensus of multiagent systems with nonlinear dynamics and sampled-data information: a delayed-input approach," International Journal of Robust and Nonlinear Control, vol. 23, no. 6, pp. 602-619, 2013.

[29] P. Lin, Y. M. Jia, and L. Li, "Distributed robust $\mathrm{H}_{\infty}$ consensus control in directed networks of agents with time-delay," Systems \& Control Letters, vol. 57, no. 8, pp. 643-653, 2008.

[30] X. Feng and W. Long, "Reaching agreement in finite time via continuous local state feedback," in Proceedings of the 26th Chinese Control Conference (CCC '07), pp. 711-715, July 2007.

[31] L. Wang and F. Xiao, "Finite-time consensus problems for networks of dynamic agents," IEEE Transactions on Automatic Control, vol. 55, no. 4, pp. 950-955, 2010.
[32] F. Xiao, L. Wang, J. Chen, and Y. Gao, "Finite-time formation control for multi-agent systems," Automatica, vol. 45, no. 11, pp. 2605-2611, 2009.

[33] Y. Zhao, Z. Duan, and G. Wen, "Distributed finite-time tracking of multiple Euler-Lagrange systems without velocity measurements," International Journal of Robust and Nonlinear Control, vol. 25, no. 11, pp. 1688-1703, 2015.

[34] H. Du, G. Wen, X. Yu, S. Li, and M. Z. Chen, "Finite-time consensus of multiple nonholonomic chained-form systems based on recursive distributed observer," Automatica, vol. 62, pp. 236242, 2015.

[35] H. Du, G. Wen, G. Chen, J. Cao, and F. E. Alsaadi, "A distributed finite-time consensus algorithm for higher-order leaderless and leader-following multiagent systems," IEEE Transactions on Systems, Man, and Cybernetics: Systems, vol. 99, pp. 1-10, 2017.

[36] C. Godsil and G. Royle, Algebraic Graph Theory, Springer, New York, NY, USA, 2001.

[37] F. Xiao, L. Wang, and Y. Jia, "Fast information sharing in networks of autonomous agents," in Proceedings of the American Control Conference (ACC '08), pp. 4388-4393, Seattle, Wash, USA, June 2008.

[38] L. Guo, Time-Varying Stochastic Systems: Stability, Estimation and Control, Jilin Science and Technology Press, 1993.

[39] S. P. Bhat and D. S. Bernstein, "Finite-time stability of continuous autonomous systems," SIAM Journal on Control and Optimization, vol. 38, no. 3, pp. 751-766, 2000.

[40] J. Cortés, "Finite-time convergent gradient flows with applications to network consensus," Automatica, vol. 42, no. 11, pp. 1993-2000, 2006. 


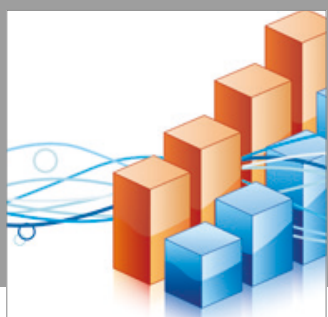

Advances in

Operations Research

vatersals

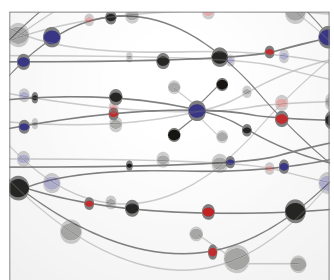

\section{The Scientific} World Journal
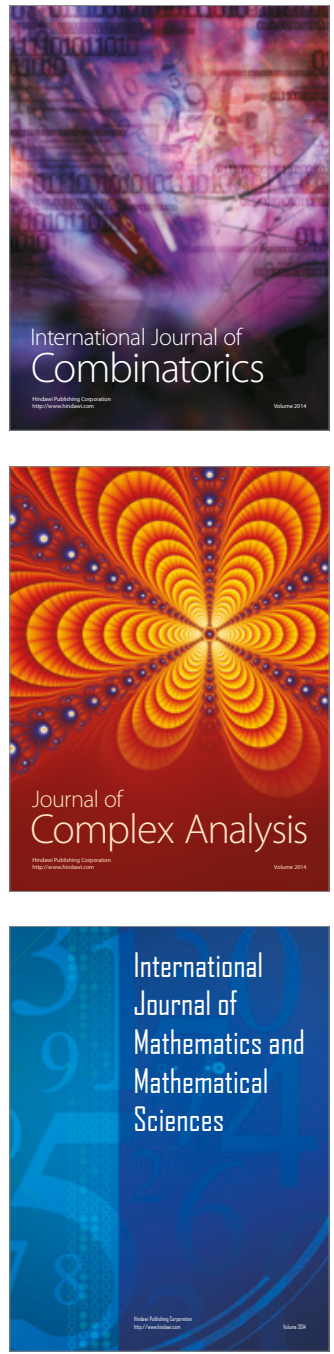
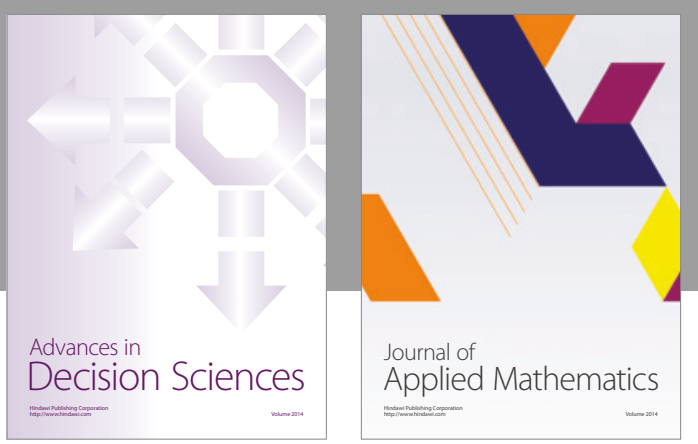

Algebra

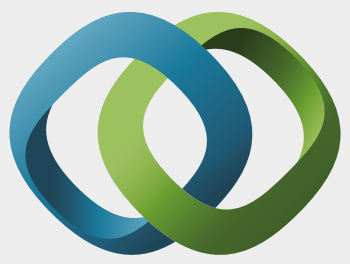

\section{Hindawi}

Submit your manuscripts at

https://www.hindawi.com
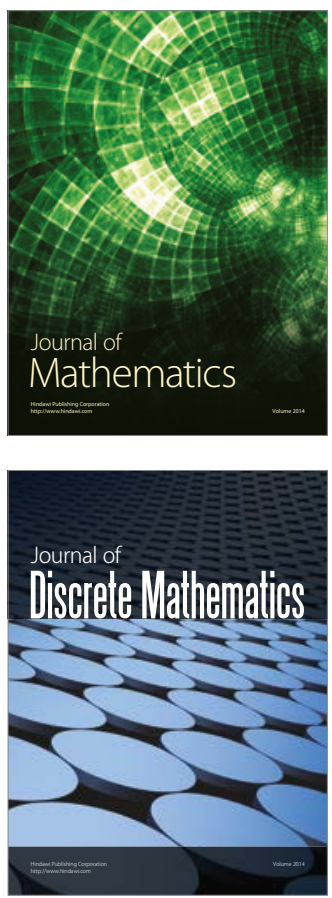

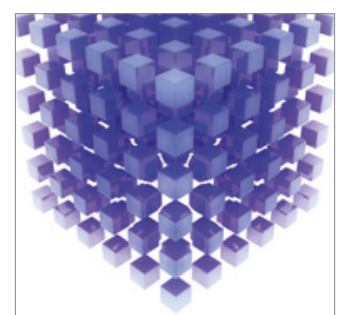

Mathematical Problems in Engineering
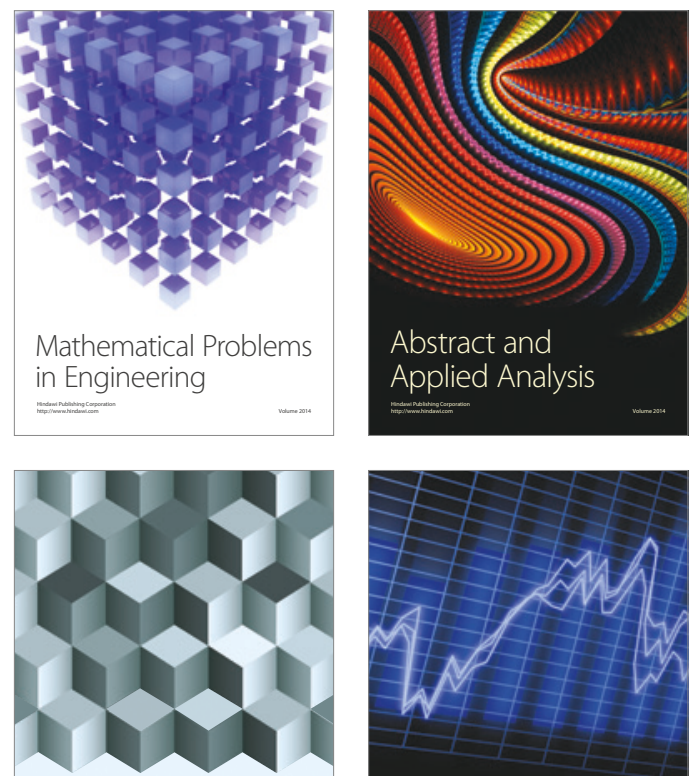

Journal of

Function Spaces

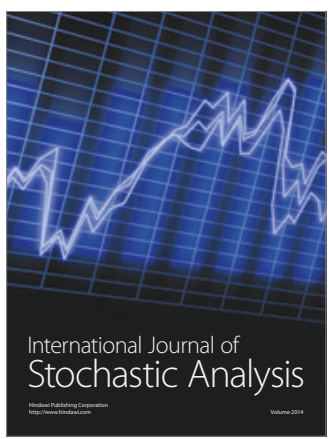

Probability and Statistics
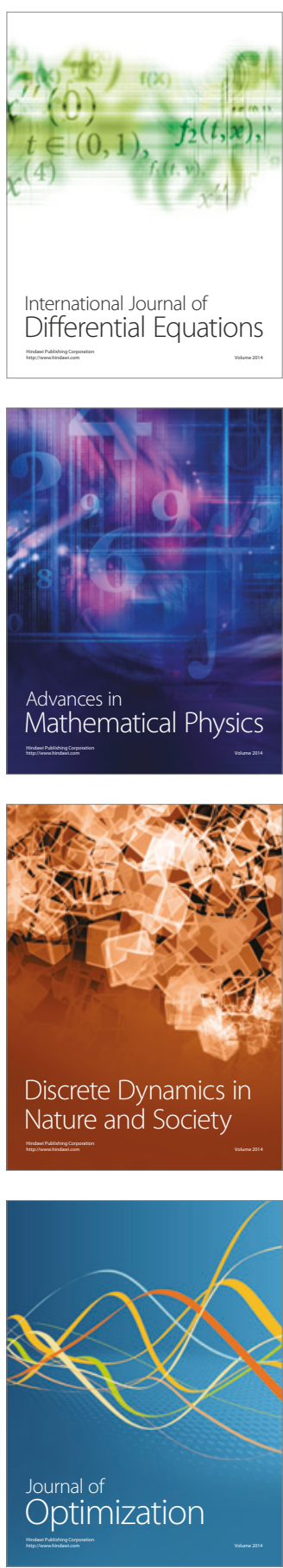